\title{
An Analytical Tire Model with Flexible Carcass for Combined Slips
}

\author{
Nan Xu, ${ }^{1}$ Konghui Guo, ${ }^{1,2}$ Xinjie Zhang, ${ }^{1,2}$ and Hamid Reza Karimi ${ }^{3}$ \\ ${ }^{1}$ State Key Laboratory of Automotive Simulation and Control, Jilin University, Changchun, Jilin 130022, China \\ ${ }^{2}$ State Key Laboratory of Advanced Design and Manufacture for Vehicle Body, Hunan University, Changsha, Hunan 410082, China \\ ${ }^{3}$ Department of Engineering, Faculty of Engineering and Science, University of Agder, 4898 Grimstad, Norway
}

Correspondence should be addressed to Nan Xu; xu.nan0612@gmail.com

Received 27 October 2013; Accepted 19 December 2013; Published 2 January 2014

Academic Editor: Xiaojie Su

Copyright (C) $2014 \mathrm{Nan} \mathrm{Xu}$ et al. This is an open access article distributed under the Creative Commons Attribution License, which permits unrestricted use, distribution, and reproduction in any medium, provided the original work is properly cited.

\begin{abstract}
The tire mechanical characteristics under combined cornering and braking/driving situations have significant effects on vehicle directional controls. The objective of this paper is to present an analytical tire model with flexible carcass for combined slip situations, which can describe tire behavior well and can also be used for studying vehicle dynamics. The tire forces and moments come mainly from the shear stress and sliding friction at the tread-road interface. In order to describe complicated tire characteristics and tire-road friction, some key factors are considered in this model: arbitrary pressure distribution; translational, bending, and twisting compliance of the carcass; dynamic friction coefficient; anisotropic stiffness properties. The analytical tire model can describe tire forces and moments accurately under combined slip conditions. Some important properties induced by flexible carcass can also be reflected. The structural parameters of a tire can be identified from tire measurements and the computational results using the analytical model show good agreement with test data.
\end{abstract}

\section{Introduction}

Tires are the only components of a road vehicle to directly contact with the road surface, and the forces and moments generated in the contact patch have significant effects on the vehicle performance [1-4]. Hence a huge number of tire models have been proposed for use in calculating the forces and moments at the tire-road interface [5-13].

The empirical models are often employed for the vehicle simulation and control, which rely basically on curve-fitted experimental data and can provide a good representation of experimental data for longitudinal force, lateral force, and aligning moment. However, empirical models would become quite complicated without theoretical support while considering all kinds of operating variables, such as slip angle, inclination angle, slip ratio, vertical load, inflation pressure, road friction, and rolling speed. The analytical models, which usually include carcass model (beam, string or rigid) with elastic tread elements, such as Brush model, Fiala model and String model, establish the relationship between tire structure parameters and tire behavior [3]. So it can be employed to derive some useful qualitative conclusions for understanding tire properties, but most of these models are either relatively simple or more complicated, which limit their practical use. In this paper, we do not see the carcass as an actual beam or string, and the carcass deformation is described with relatively simple and general forms, composed by translational, bending, and twisting deformations. Besides, the arbitrary pressure distribution, dynamic friction coefficient, and anisotropic stiffness properties are also considered. Consequently, the analytical model would become more suitable for application and also appropriate for analyzing tire properties in detail.

In this paper, the key factors for developing the analytical tire model are firstly discussed; then, considering all these factors, the analytical tire model with flexible carcass for combined slips is introduced. By employing the model, effects of carcass compliance on tire properties are discussed, which are valuable for understanding tire properties; at the end, the analytical model is validated by test data. 


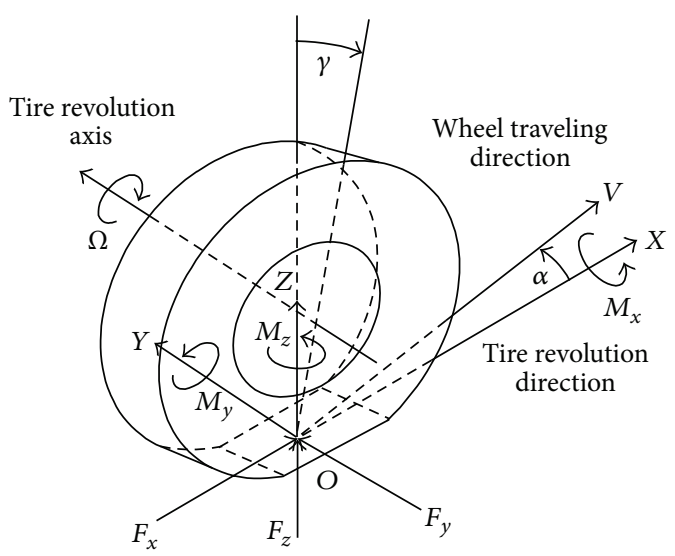

FIGURE 1: Tire axis system.

\section{Tire Axis System and Slip Ratios}

The tire axis system is shown in Figure 1. The positive direction of the $X$-axis and the $Y$-axis is coincident with tire revolution direction (not wheel traveling direction); the $Z$ axis is perpendicular to road plane and upward. The wheel traveling speed is denoted as $V, \alpha$ is the slip angle, and $\gamma$ is inclination angle. The figure shows all the forces and moments associated with a wheel.

The longitudinal and lateral slip ratios are defined in the unified form [11]

$$
\begin{gathered}
S_{x}=\frac{-V_{s x}}{\Omega R_{e}}=-\frac{V \cos \alpha-\Omega R_{e}}{\Omega R_{e}} \quad S_{x} \in(-\infty,+\infty), \\
S_{y}=\frac{-V_{s y}}{\Omega R_{e}}=-\frac{V \sin \alpha}{\Omega R_{e}} \quad S_{y} \in(-\infty,+\infty),
\end{gathered}
$$

where $\Omega$ is the angular speed, $R_{e}$ is the effective rolling radius and $V_{s x}$ and $V_{s y}$ are the longitudinal and lateral sliding speeds of tire with respect to road surface.

Usually, the longitudinal slip ratio used in tire force test is defined as

$$
\kappa=\frac{-V_{s x}}{V \cos \alpha} .
$$

The relationship between $S_{x}$ and $\kappa$ can be obtained easily:

$$
S_{x}=\frac{\kappa}{1+\kappa}
$$

\section{Key Factors for Analytical Tire Model}

3.1. Arbitrary Pressure Distribution. Contact pressure distribution over contact patch strongly influences tire behaviors. Here, the contact patch is assumed to be rectangular in shape, and the contact pressure distribution is assumed to be uniform in lateral direction and of arbitrary form in circumferential direction to represent different kinds of pressure distributions.
The contact pressure $q_{z}$ along the contact patch length $2 a$ is expressed as an arbitrary form as

$$
q_{z}(x)=\frac{F_{z}}{2 a} \cdot \eta(u),
$$

where $F_{z}$ is the tire vertical load; $u=x / a$ is the relative coordinate. $\eta(u)$ is the normalized pressure distribution function and is expressed as

$$
\begin{gathered}
\eta(u)=A \cdot\left(1-u^{2 n}\right) \cdot\left(1+\lambda \cdot u^{2 n}\right) \cdot(1-B \cdot u) \\
A=\frac{(2 n+1)(4 n+1)}{2 n(4 n+1+\lambda)}, \\
B=-\frac{3(2 n+3)(4 n+3)(4 n+1+\lambda)}{(2 n+1)(4 n+1)(4 n+3+3 \lambda)} \cdot \frac{\Delta}{a},
\end{gathered}
$$

where $n, \lambda$, and $\Delta$ are the parameters which determine the shape of pressure distribution. With these three parameters, (5) can be employed to express arbitrary pressure distribution over contact patch, as shown in Figure 2.

3.2. Carcass Structure Parameters. The deformation of carcass has an important effect on the tire properties under combined slip conditions. In this paper, three tire carcass stiffness parameters for tire lateral carcass deformation are introduced, that is, tire carcass lateral translation stiffness $K_{c y}$, carcass bending stiffness $K_{c b}$, and carcass twisting stiffness $N_{\theta}$. So, the carcass deformation includes lateral translating part $y_{c 0}$, bending part $y_{c b}$, and twisting part $y_{\theta}$, as shown in Figure 3 . The carcass longitudinal deformation is assumed to include longitudinal translating part $x_{c 0}$, and the longitudinal translation stiffness is $K_{c x}$.

The lateral translating deformation of carcass can be calculated as

$$
y_{c 0}=\frac{F_{y}}{K_{c y 0}},
$$

where $K_{c y}$ is the lateral translation stiffness of carcass; $F_{y}$ is the lateral force.

The lateral bending deformation of carcass can be calculated as

$$
y_{c b}(x)=\frac{F_{y}}{K_{c b}} \cdot \xi\left(\frac{x}{a}\right),
$$

where $K_{c b}$ is the carcass bending stiffness; $\xi(x / a)$ is the general function of carcass bending deformation.

The zero-order moment and first-order moment of $\xi(u)$ are expressed as

$$
\begin{gathered}
D_{0}(u)=\int_{u}^{1} \xi(u) d u, \\
D_{1}(u)=\int_{u}^{1} u \cdot \xi(u) d u .
\end{gathered}
$$

The twisting deformation of carcass is calculated by

$$
\begin{gathered}
y_{\theta}(x)=\theta \cdot x, \\
\theta=\frac{M_{z}}{N_{\theta}},
\end{gathered}
$$




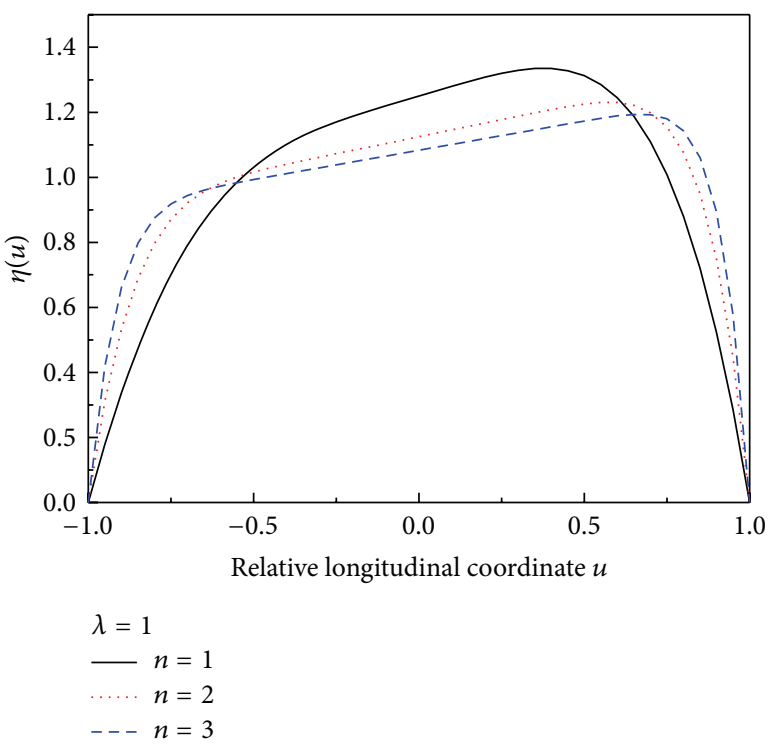

(a)

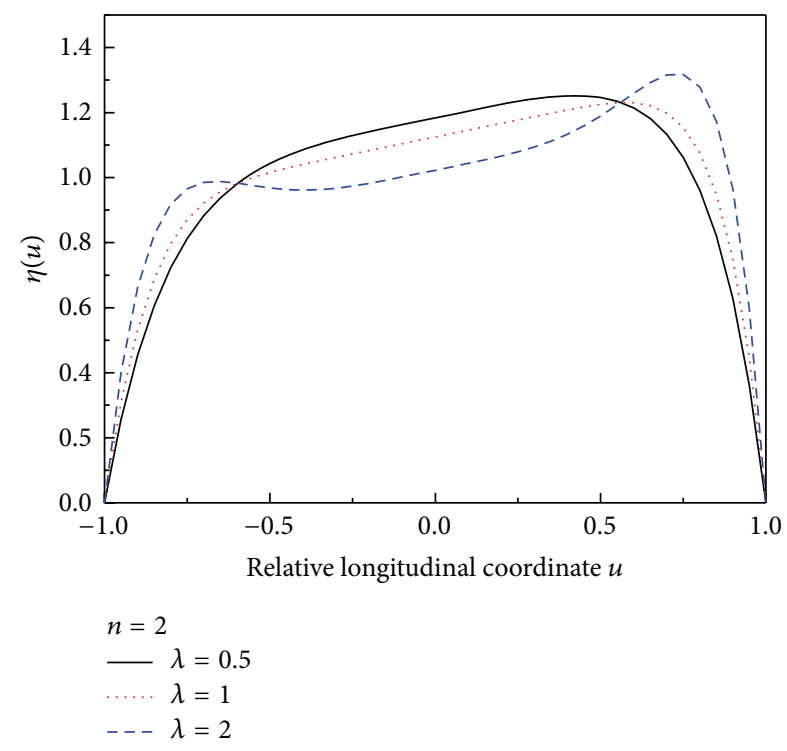

(b)

FIgURE 2: Pressure distribution.

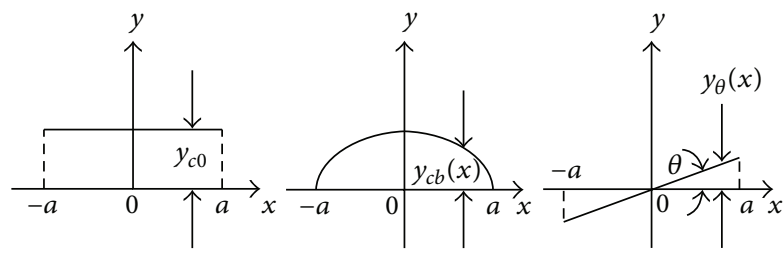

FIGURE 3: Tire lateral carcass deformation.

where $\theta$ is the carcass twisting angle; $N_{\theta}$ is the carcass twisting stiffness; $M_{z}$ is the aligning moment.

The longitudinal translating deformation of carcass is expressed as

$$
x_{c 0}=\frac{F_{x}}{K_{c x 0}}
$$

where $K_{c x 0}$ is the longitudinal translation stiffness of carcass; $F_{x}$ is the longitudinal force.

3.3. Dynamic Friction Coefficient. Friction coefficient used in the analytical tire model is the dynamic friction coefficient, which considers the significant influence of slip speed. The expression is as follows [14]:

$$
\begin{aligned}
& \mu_{d} \\
& =\mu_{s}+\left(\mu_{m}-\mu_{s}\right) \\
& \quad \cdot \exp \left(-\mu_{h}^{2} \cdot \log ^{2}\left(\left|\frac{V_{s}}{V_{s m}}\right|+N \cdot \exp \left(-\left|\frac{V_{s}}{V_{s m}}\right|\right)\right)\right),
\end{aligned}
$$

where $\mu_{0}, \mu_{s}, \mu_{h}$, and $V_{s m}$ are friction characteristic parameters; $N$ (usually $N=0.8$ ) is a factor to make the friction coefficient increase slightly around the origin; $V_{s}$ is the slip speed between the road and tire.
3.4. Anisotropic Stiffness Properties. The anisotropy of tire slip stiffness will arouse the difference of tire shear stress direction in adhesion region and sliding region, which are expressed as [15]

$$
\begin{gathered}
\text { adhesion : } \theta_{\mathrm{ad}}=\arctan \left(\frac{\left(K_{y} S_{y}\right)}{\left(K_{x} S_{x}\right)}\right), \\
\text { sliding }: \theta_{s}=\arctan \left(\frac{S_{y}}{S_{x}}\right),
\end{gathered}
$$

where $K_{x}$ and $K_{y}$ represent the longitudinal slip and cornering stiffness, respectively.

\section{Analytical Tire Forces and Moments Model}

4.1. Tire Forces and Moments without Sliding. In order to obtain the shear force in the contact patch, the deformations of the tread and carcass along the $X$ and $Y$ axes must be known firstly. The longitudinal and lateral deformations of the tread and carcass, under combined slip condition, are shown in Figure 4. In this figure, $X O Y$ is a coordinate system before the carcass is deformed, and xoy is a relative coordinate system for describing the tread deformation and bending and twisting deformation of carcass. $x_{c 0}$ and $y_{c 0}$ are the longitudinal and lateral translating deformation of carcass. "ABC" is the contact line of the contact patch under combined slip condition. $V$ is the wheel traveling speed; $\alpha$ is the slip angle and $\theta$ is the carcass twisting angle. In general case, the whole length of contact patch, $2 a$, is divided into two parts, the adhesion region " $\mathrm{AB}$ " and the sliding region " $\mathrm{BC}$," by the initial sliding point "B." 


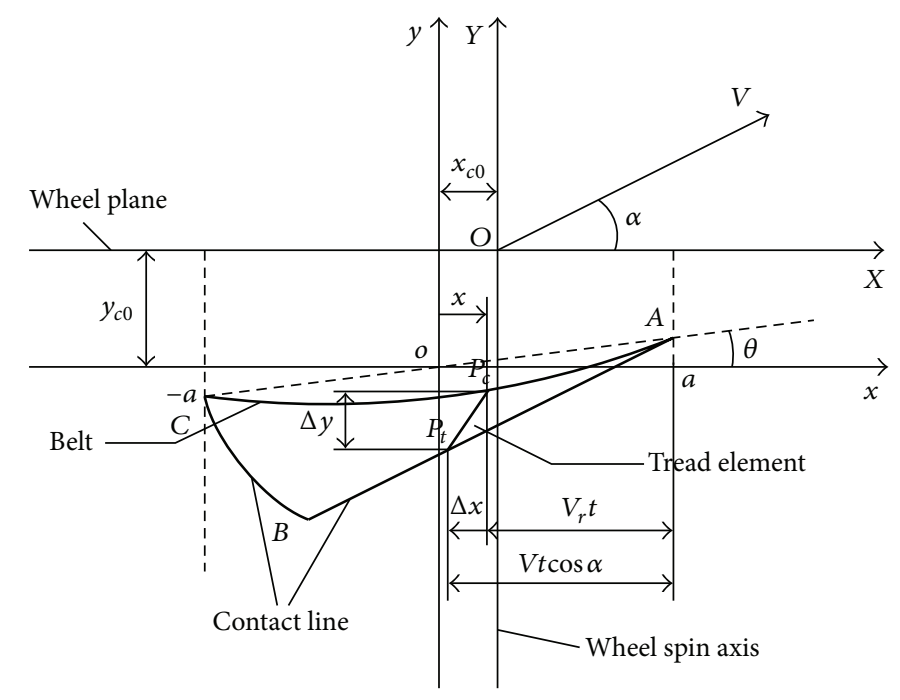

FiguRE 4: Deformation of carcass and tread element under combined slip condition.

$P_{c} P_{t}$ in the figure represents the tread element after rolling for a period of time $t$. The upper point of tread element, $P_{c}$, is attached to the belt of tire. Its coordinate could be written as

$$
\begin{gathered}
x_{p c}=x \\
y_{p c}=y_{\theta}(x)+y_{c b}(x) .
\end{gathered}
$$

The lower point of tread element, $P_{t}$, is contacted with the ground. Its coordinate could be written as

$$
\begin{gathered}
x_{p t}=x-\left(V t \cos \alpha-V_{r} t\right)=x+(a-x) S_{x}, \\
y_{p t}=-V t \sin \alpha+a \theta=a \theta+(a-x) S_{y} .
\end{gathered}
$$

Therefore, the longitudinal and lateral deformations of tread element is

$$
\begin{gathered}
\Delta x=x_{p t}-x_{p c}=(a-x) S_{x} \\
\Delta y=y_{p t}-y_{p c}=(a-x)\left(\frac{M_{z}}{N_{\theta}}+S_{y}\right)-\frac{F_{y}}{K_{c b}} \xi\left(\frac{x}{a}\right) .
\end{gathered}
$$

The main source of anisotropy is due to different tire structural flexibility in lateral and longitudinal direction, whereas tread anisotropy is present but comparatively small. In this paper, the stiffness of tread, denoted as $k_{t}$, is considered to be isotropic. The shear stresses of tread element in $X$ and $Y$ directions can be expressed as

$$
\begin{gathered}
q_{x}=k_{t} \cdot \Delta x=k_{t} \cdot(a-x) S_{x} \\
q_{y}=k_{t} \cdot \Delta y=k_{t} \cdot\left[(a-x)\left(\frac{M_{z}}{N_{\theta}}+S_{y}\right)-\frac{F_{y}}{K_{c b}} \xi\left(\frac{x}{a}\right)\right] .
\end{gathered}
$$

The shear force in the directions $X$ and $Y$ can be determined as follows:

$$
\begin{gathered}
F_{x}=\int_{-a}^{a} q_{x} d x \\
F_{y}=\int_{-a}^{a} q_{y} d x \\
M_{z}=-\int_{-a}^{a} q_{x} \cdot y_{p c} d x+\int_{-a}^{a} q_{y} \cdot x_{p c} d x-F_{x} \cdot y_{c 0}+F_{y} \cdot x_{c 0} .
\end{gathered}
$$

Considering the previous equations (6) (10), (13), and (16), the forces and moments could be written as

$$
\begin{gathered}
F_{x}=2 a^{2} k_{t} S_{x}, \\
F_{y}=\frac{3}{a} \varepsilon_{\theta} M_{z}+2 a^{2} k_{t} S_{y}-\varepsilon_{b} F_{y} \\
M_{z}=-a \varepsilon_{b} S_{x} F_{y}+\varepsilon_{\theta} S_{x} M_{z}+\frac{1}{2} a \varepsilon_{b} S_{x} F_{y} D_{1}(-1)-\varepsilon_{\theta} M_{z} \\
-\frac{2}{3} a^{3} k_{t} S_{y}-\frac{1}{2} a \varepsilon_{b} F_{y} D_{1}(-1)+\left(\frac{1}{K_{c x 0}}-\frac{1}{K_{c y 0}}\right) F_{x} F_{y},
\end{gathered}
$$

where the bending characteristic ratio and twisting characteristic ratio have been introduced and defined by

$$
\begin{gathered}
\varepsilon_{b}=\frac{2 a k_{t}}{K_{c b}} \\
\varepsilon_{\theta}=\frac{2}{3} \frac{a^{3} k_{t}}{N_{\theta}} .
\end{gathered}
$$


Solving (18) to obtain the explicit expression of $F_{y}$ and $M_{z}$, (18) becomes

$$
\begin{gathered}
F_{x}=K_{x} S_{x}, \\
F_{y}=K_{y} S_{y}, \\
M_{z}=K_{m} S_{y},
\end{gathered}
$$

with

$$
\begin{gathered}
K_{x}=2 a^{2} k_{t}, \\
K_{y}=\frac{a\left(1+\varepsilon_{\theta}-\varepsilon_{\theta} S_{x}\right) K_{y 0}-3 \varepsilon_{\theta} K_{m 0}}{a\left(1+\varepsilon_{b}\right)\left(1+\varepsilon_{\theta}-\varepsilon_{\theta} S_{x}\right)+3 a \varepsilon_{b} \varepsilon_{\theta} S_{x}-3 \varepsilon_{\theta} c F_{x}} \\
K_{m}=\frac{a}{3 \varepsilon_{\theta}}\left[\left(1+\varepsilon_{b}\right) K_{y}-K_{y 0}\right]
\end{gathered}
$$

where $K_{x}$ is the longitudinal slip stiffness, $K_{y}$ is the cornering stiffness, and $K_{m}$ is the aligning stiffness with flexible carcass. $K_{y 0}$ and $K_{m 0}$ are the cornering stiffness and aligning stiffness, respectively, when the carcass is assumed to be rigid, which are expressed as

$$
K_{y 0}=2 a^{2} k_{t}, \quad K_{m 0}=\frac{2}{3} a^{3} k_{t}
$$

where $c$ in (21) is the translating compliance coefficient that is defined by

$$
c=\frac{1}{K_{c x 0}}-\frac{1}{K_{c y 0}} .
$$

4.2. Tire Forces and Moments in General Case with Sliding Region. Considering that the sliding region might exist, the shear force in this area should be expressed as $q(x)=\mu q_{z}(x)$, based on which the coordinate of initial sliding point, $x=x_{c}$, or the relative coordinate $u_{c}=x_{c} / a$, can be solved. The coordinate of initial sliding point will satisfy the following equation:

$$
q=\sqrt{q_{x}^{2}+q_{y}^{2}}=\frac{\mu F_{z}}{2 a} \cdot \eta\left(u_{c}\right) .
$$

Integrating through both adhesion and sliding regions, the tire forces and moments can be derived as

$$
\begin{aligned}
F_{x}= & \int_{x_{c}}^{a} k_{t} \cdot \Delta x d x+\int_{-a}^{x_{c}} \frac{\mu F_{z}}{2 a} \cdot \eta\left(\frac{x}{a}\right) \cdot \theta_{s x} d x, \\
F_{y}= & \int_{x_{c}}^{a} k_{t} \cdot \Delta y d x+\int_{-a}^{x_{c}} \frac{\mu F_{z}}{2 a} \cdot \eta\left(\frac{x}{a}\right) \cdot \theta_{s y} d x, \\
M_{z}= & -\int_{x_{c}}^{a} k_{t} \cdot \Delta x \cdot y_{p c} d x+\int_{x_{c}}^{a} k_{t} \cdot \Delta y \cdot x_{p c} d x \\
& -\int_{-a}^{x_{c}} \frac{\mu F_{z}}{2 a} \cdot \eta\left(\frac{x}{a}\right) \cdot \theta_{s x} \cdot y_{p c} d x \\
& +\int_{-a}^{x_{c}} \frac{\mu F_{z}}{2 a} \cdot \eta\left(\frac{x}{a}\right) \cdot \theta_{s y} \cdot x_{p c} d x \\
& -F_{x} \cdot y_{c 0}+F_{y} \cdot x_{c 0},
\end{aligned}
$$

where $\theta_{s x}$ and $\theta_{s y}$ are, respectively, the longitudinal and lateral components of shear stress direction $\theta_{s}$ in sliding region.

Substituted with (6) (12), (13), and (15), (25) becomes

$$
\begin{gathered}
F_{x}=B_{6} \cdot K_{x} S_{x}+B_{3} \cdot \mu F_{z} \theta_{s x} \\
F_{y}=\left[\left(P_{1} B_{6} \cdot K_{y 0}+P_{4} B_{7} \cdot K_{m 0}\right) S_{y}\right. \\
\left.\quad+\left(P_{1} B_{3}+P_{4} B_{4} a\right) \mu F_{z} \theta_{s y}\right] \\
\quad \times\left(P_{2} P_{4}+P_{1} P_{3}\right)^{-1}, \\
M_{z}=\left[\left(-P_{2} B_{6} \cdot K_{y 0}+P_{3} B_{7} \cdot K_{m 0}\right) S_{y}\right. \\
\left.\quad+\left(-P_{2} B_{3}+P_{3} B_{4} a\right) \mu F_{z} \theta_{s y}\right] \\
\times \\
\quad\left(P_{2} P_{4}+P_{1} P_{3}\right)^{-1},
\end{gathered}
$$

with

$$
\begin{gathered}
B_{1}=\frac{1}{2} \varepsilon_{b} D_{0}\left(u_{c}\right), \quad B_{2}=\frac{1}{2} \varepsilon_{b} D_{1}\left(u_{c}\right), \\
B_{3}=\frac{1}{2} m_{0}\left(u_{c}\right), \\
B_{4}=\frac{1}{2} m_{1}\left(u_{c}\right), \quad B_{5}=\frac{1}{2} m_{D}\left(u_{c}\right), \\
B_{6}=\frac{\left(1-u_{c}\right)^{2}}{4}, \quad B_{7}=\frac{1}{4}\left(1-3 u_{c}^{2}+2 u_{c}^{3}\right), \\
P_{1}=1-B_{7} \varepsilon_{\theta}+B_{7} \varepsilon_{\theta} S_{x}+B_{4} \mu F_{z} \theta_{s x} a \frac{1}{N_{\theta}}, \\
P_{2}=\left(B_{2}+B_{1} S_{x}-B_{2} S_{x}\right) a+B_{5} \mu F_{z} \theta_{s x} \frac{1}{K_{c b}}-c F_{x}, \\
P_{3}=1+B_{1}, \quad P_{4}=\frac{3}{a} B_{6} \varepsilon_{\theta},
\end{gathered}
$$

where $m_{0}\left(u_{c}\right), m_{1}\left(u_{c}\right)$, and $m_{D}\left(u_{c}\right)$ are defined by

$$
\begin{gathered}
m_{0}\left(u_{c}\right)=\int_{-1}^{u_{c}} \eta(u) d u, \quad m_{1}\left(u_{c}\right)=\int_{-1}^{u_{c}} u \eta(u) d u, \\
m_{D}\left(u_{c}\right)=\int_{-1}^{u_{c}} \xi(u) \eta(u) d u .
\end{gathered}
$$

\section{Simulation Analysis and Experiment Validation}

According to the analytical tire model, the tire forces and moments under combined slip conditions can be simulated and the effects of carcass structure parameters can be analyzed. Furthermore, the analytical tire model can also be used in vehicle dynamics simulation by identifying the model parameters with test data. 


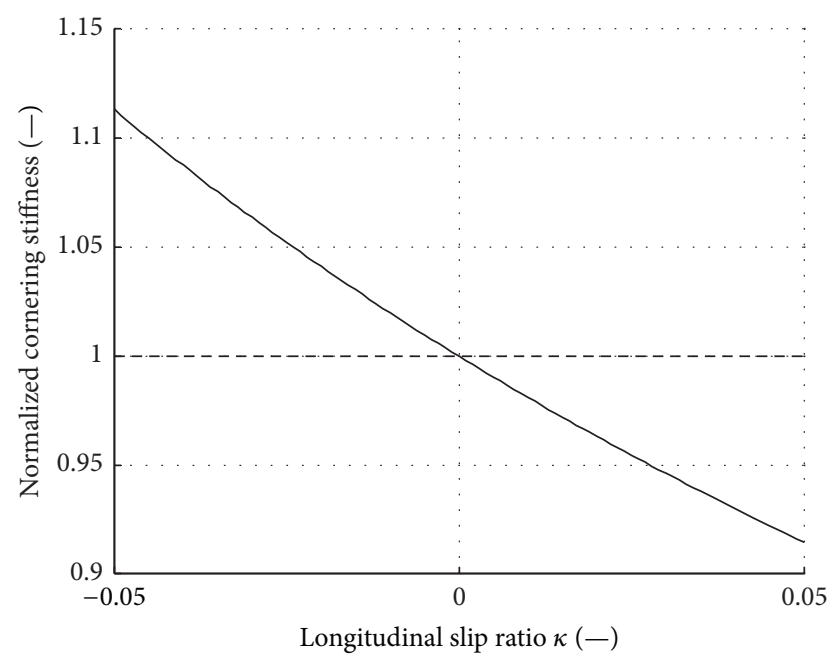

FIGURE 5: Variation of cornering stiffness under combined slip condition.

5.1. Effect of Carcass Flexible on Tire Cornering Stiffness under Combined Slip Condition. First of all, the cornering stiffness under pure side slip condition could be calculated by assuming $S_{x}=0$ in (21),

$$
K_{y \text { pure }}=K_{y 0} \cdot \frac{1}{\left(1+\varepsilon_{b}\right)\left(1+\varepsilon_{\theta}\right)} .
$$

It can be seen that the bending characteristic ratio $\varepsilon_{b}$ and the twisting characteristic ratio $\varepsilon_{\theta}$ have important influence on cornering stiffness. With the increase of $\varepsilon_{b}$ or $\varepsilon_{\theta}$, the cornering stiffness will decrease obviously.

Then, the variation of cornering stiffness under combined slip condition is also our concern, which often has important influence on tire characteristics. Divided by $K_{y \text { pure }}$, the normalized cornering stiffness could be derived as

$$
\frac{K_{y}}{K_{y \text { pure }}}=\frac{\left(1-\varepsilon_{\theta} S_{x}\right)\left(1+\varepsilon_{b}\right)\left(1+\varepsilon_{\theta}\right)}{\left(1+\varepsilon_{b}\right)\left(1+\varepsilon_{\theta}-\varepsilon_{\theta} S_{x}\right)+3 \varepsilon_{b} \varepsilon_{\theta} S_{x}-(3 / a) \cdot \varepsilon_{\theta} c F_{x}} .
$$

It can be seen that the normalized cornering stiffness is a function of $S_{x}$, which means that the longitudinal slip ratio (or longitudinal force) will have influence on the tire cornering stiffness. Figure 5 shows the relationship between the normalized cornering stiffness and longitudinal slip ratio. It indicates that the cornering stiffness will increase with the action of braking force does not and decrease when acting driving force.

\subsection{Effect of Carcass Compliance on Aligning Moment under} Combined Slip Condition. The carcass translating compliance has a significant influence on aligning moment for a braked or driven wheel. In Figure 6, $c$ is the translating compliance coefficient defined in (23). As shown in Figure 6, the curve becomes more and more asymmetric when $c$ increases (absolute value) and $M_{z}$ changes its sign in the braking half of

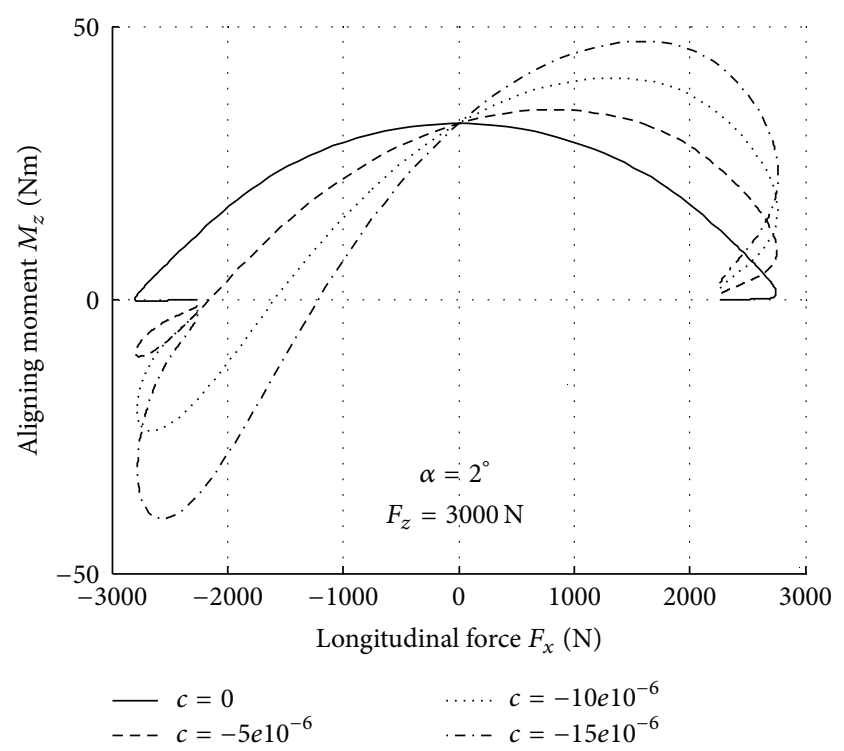

FIGURE 6: Influence of carcass compliance on aligning moment under combined slip condition.

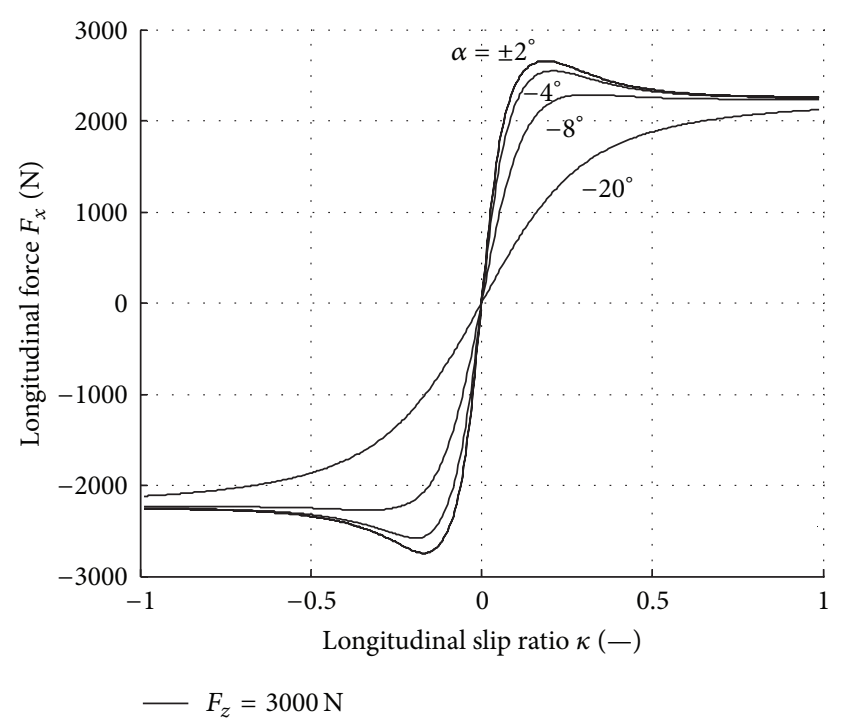

FIgURE 7: Longitudinal force characteristics.

the diagram. These phenomena correspond reasonably well with the experimental results given in the previous scholars' researches [2].

5.3. Simulation Results of Tire Forces and Moments under Combined Slip Condition. In this part, a number of example results of using the analytical tire model have been presented, as shown in Figures 7-11. Some important characteristics have been reflected.

(1) Dynamic friction coefficient: Figure 7 shows the longitudinal force under combined slip conditions. With the increase of slip angle $\alpha$, the longitudinal force will decrease because of the limitation of friction 


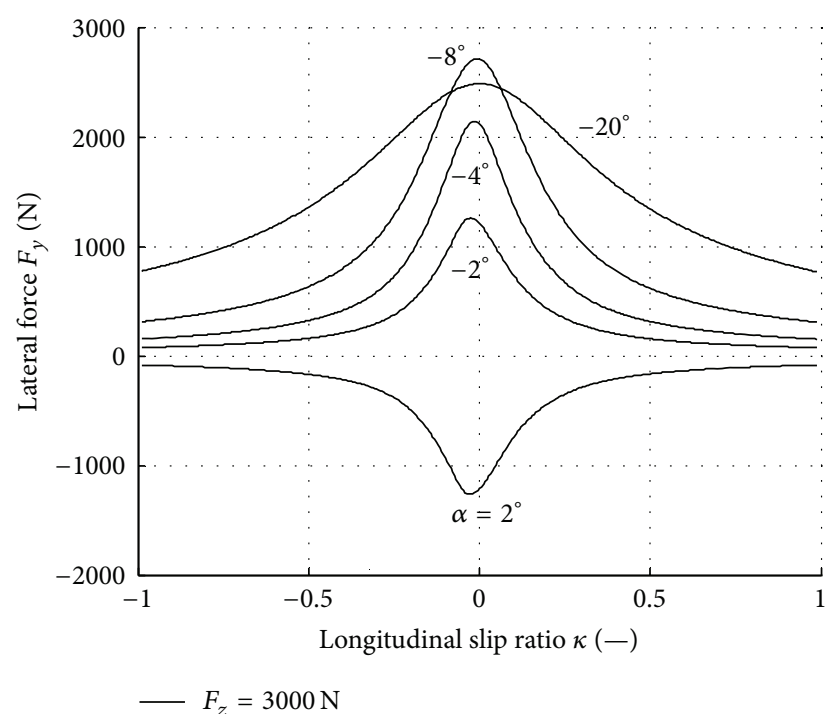

FIGURE 8: Lateral force characteristics.

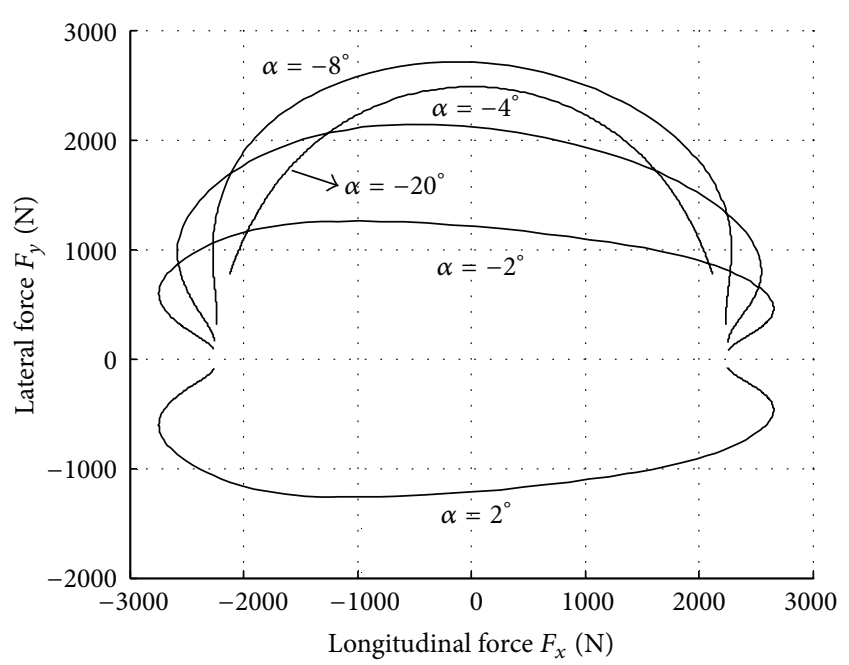

$-F_{z}=3000 \mathrm{~N}$

FIGURE 9: $F_{y}$ versus $F_{x}$ characteristics.

force between the tire and the road. More important, because we adopt the dynamic friction coefficient as shown in (11), the sliding velocity dependent friction coefficient can be seen obviously in Figure 7.

(2) The variation of cornering stiffness: Figure 8 shows the lateral force for combined slip and Figure 9 shows the $F_{y}-F_{x}$ characteristics, which indicate the decrease of lateral force with the increase of longitudinal slip ratio. Besides, it can be seen that the lateral force has an increase to some extent when the wheel is braked slightly, which is mainly due to the increase of cornering stiffness.

(3) The asymmetry of aligning moment: Figure 10 shows the aligning moment for combined slip. It is obvious

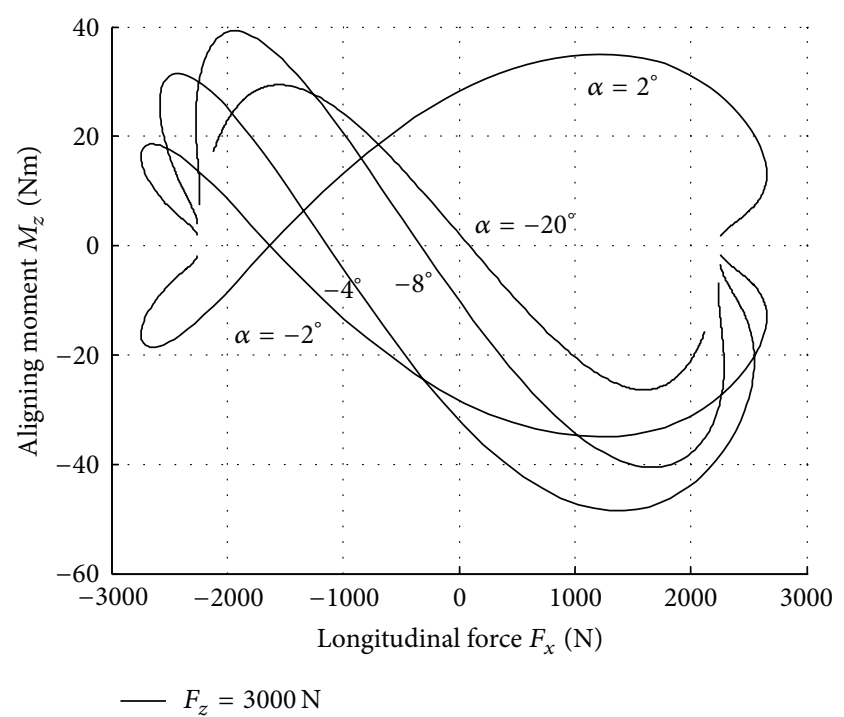

FIGURE 10: Aligning moment characteristics $\left(M_{z}\right.$ versus $\left.F_{x}\right)$.

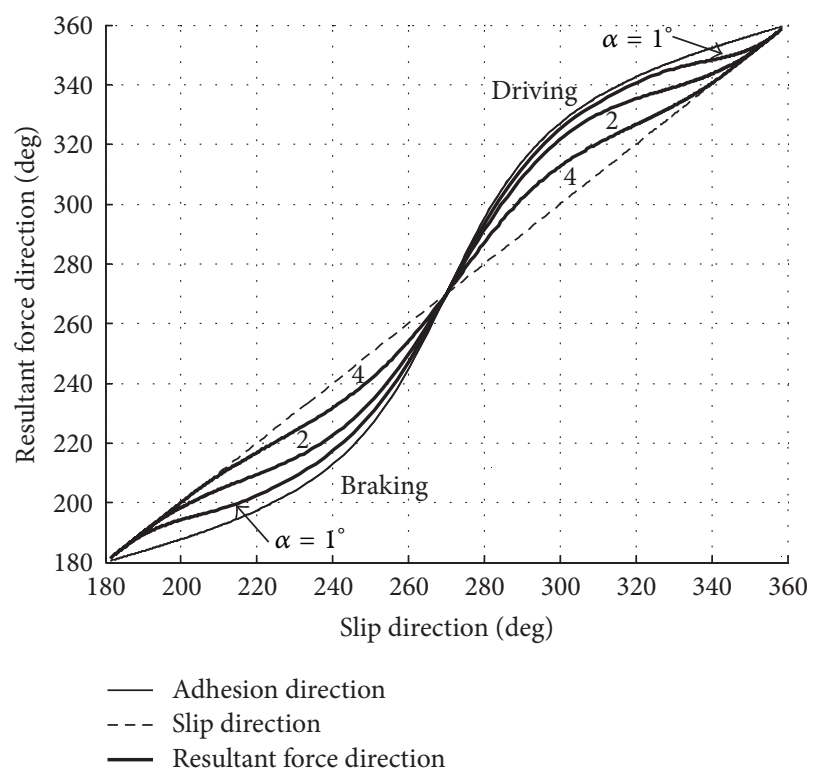

FIGURE 11: Resultant force direction.

that the curve is asymmetric and $M_{z}$ changes its sign in the braking half of the diagram.

(4) Anisotropic stiffness properties: the anisotropy of tire slip stiffness will cause the variation of resultant force direction under different combined slip conditions. Figure 11 shows the relationship between the resultant force direction and the slip direction. It can be seen that the resultant force direction changes gradually from adhesion direction to slip direction, which is reasonably and coincides with the test data provided in [15].

Some simulated results using the Brush model are shown in Figures 12 and 13. Figure 12 is the $F_{y}-F_{x}$ characteristics with different slip angles under combined slip conditions, and 


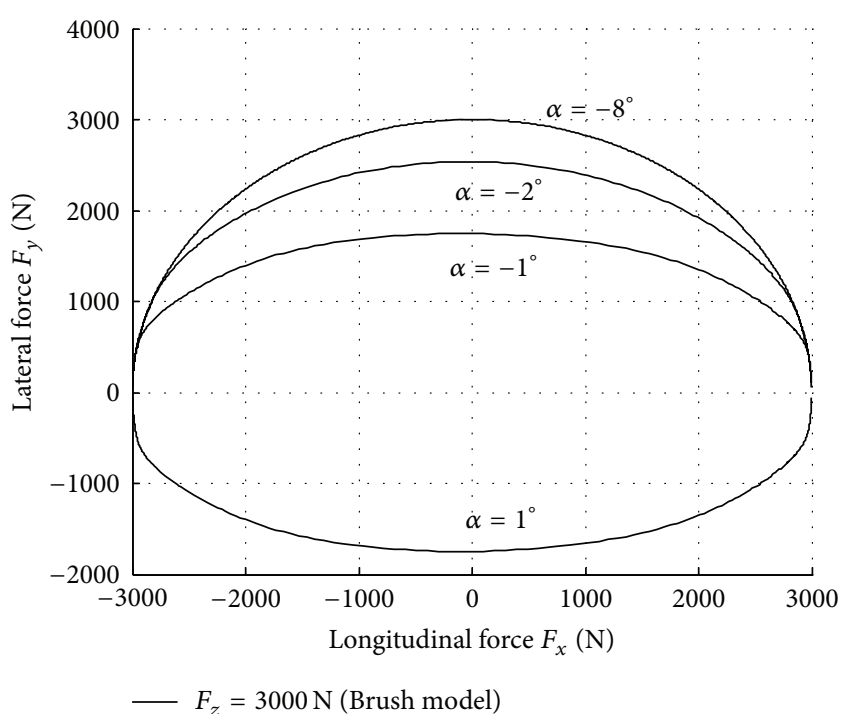

FIGURE 12: $F_{y}$ versus $F_{x}$ characteristics (Brush model).

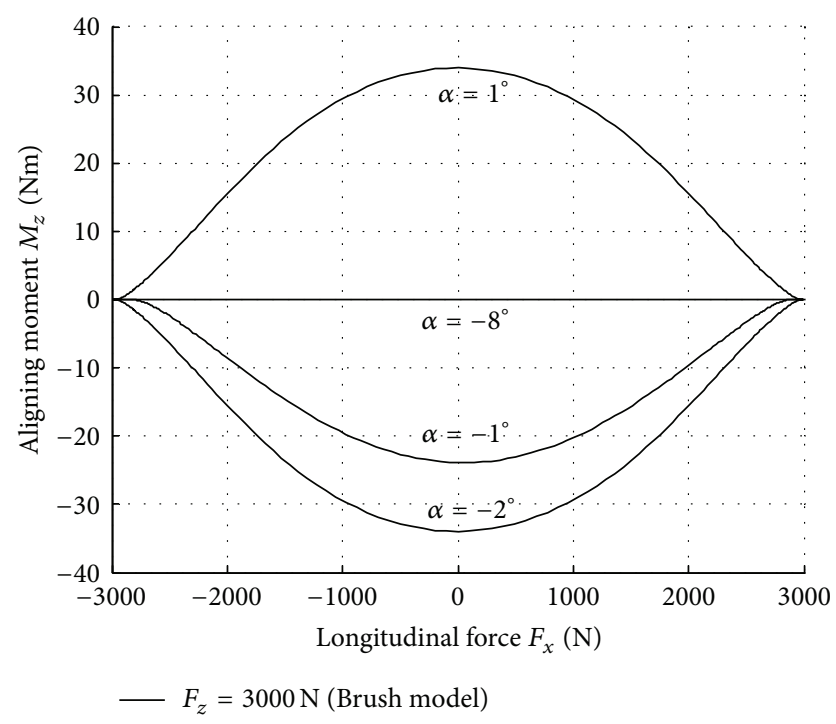

Figure 13: $M_{z}$ versus $F_{x}$ characteristics (Brush model).

Figure 13 shows the $M_{z}-F_{x}$ characteristics. Comparing the results of analytical tire model with flexible carcass proposed in this paper, it is obvious that the Brush model have the capacity to express the declining friction coefficient and the influence of carcass flexibility, which will lead to severe deviation for tire characteristics, especially for the aligning moments. The dynamic friction coefficient will cause the curve of $F_{y}-F_{x}$ characteristics to turn inward, as shown in Figure 9. The flexible carcass will make the curve of $M_{z}-F_{x}$ characteristics asymmetric, as shown in Figure 10.

5.4. Experiment Validation. Test data for the P245/65R 17 specification at different slip angles are used to validate the analytical tire model. Figure 14 shows the tire shear forces under combined slip conditions. The vertical load is $4000 \mathrm{~N}$

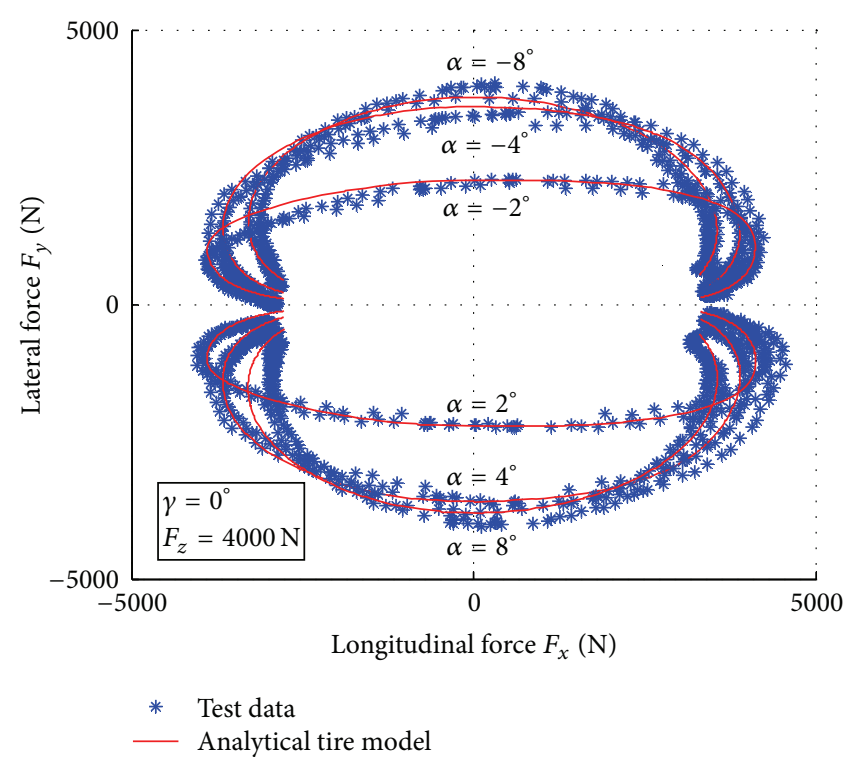

FIGURE 14: The comparison between the analytical tire model and test data.

and both the longitudinal and lateral forces are tested when the tire is rolling under combined tire cornering and braking conditions. From Figure 14, it can be seen that the analytical tire model can describe the tire characteristics accurately under combined slip conditions.

\section{Conclusions}

This paper presented an analytical tire model with flexible carcass for combined slips. With employing the model, the effects of carcass structure parameters on tire properties are discussed. The simulation results and experiment validation of longitudinal forces, lateral forces, and aligning moments under combined slip conditions are also provided. Some conclusions are summarized as follows.

Firstly, arbitrary pressure distribution, translational, bending, and twisting compliance of the carcass, dynamic friction coefficient, and anisotropic stiffness properties are the key factors for developing the analytical tire model.

Secondly, the carcass compliance has a great influence on tire cornering stiffness. With the increase of bending characteristic ratio $\varepsilon_{b}$ or twisting characteristic ratio $\varepsilon_{\theta}$, the cornering stiffness will decrease obviously. Moreover, the cornering stiffness is also influenced by the braking force or driving force and thus leads to the increase of lateral force when tire has a slight braking.

Thirdly, the carcass translating compliance has a significant influence on aligning moment under combined slip conditions. The curve of $M_{z}$ becomes more and more asymmetric when translating compliance coefficient $c$ increases (absolute value) and $M_{z}$ changes its sign in the braking half of the diagram.

Fourthly, the dynamic friction coefficient can express the friction delay with sliding velocity effectively; the anisotropic stiffness properties can express the resultant force direction 
reasonably, which changes gradually from adhesion direction to slip direction.

Finally, considering all these key factors, the analytical tire model is capable of describing all kinds of tire properties reasonably and accurately. The model parameters can also be identified from tire measurements and the computational results using the analytical model show good agreement with test data.

\section{Conflict of Interests}

The authors declare that there is no conflict of interests regarding the publication of this paper.

\section{Acknowledgments}

The authors would like to thank the previous joint project between the Research and Development Center of General Motors and the State Key Laboratory of Automotive Simulation and Control at Jilin University, from which the test data presented in this paper are produced. Special thanks are due to the Open Research Fund Program of the State Key Laboratory of Advanced Design and Manufacturing for Vehicle Body (31115028), the China Postdoctoral Science Foundation (2012M520028), the National Natural Science Foundation of China (51205155), and the National Basic Research Program of China (973 Program) (2011CB711201) for supporting authors' research. The authors also thank the staff in the State Key Laboratory of Automobile Dynamics Simulation for their contribution to this study.

\section{References}

[1] K. H. Guo, Handling Dynamics of Automobiles, Jilin Press of Science and Technology, 1991, Chinese.

[2] J. C. Dixon, Tires, Suspension and Handling, The SAE Publications Group, Warrendale, Pa, USA, 2nd edition, 1996.

[3] H. B. Pacejka, Tyre and Vehicle Dynamics, Butterworth Heinemann, 2002.

[4] C. Z. Yin, L. Bu, and H. T. Gong, "Mathematical model and algorithm of split load vehicle routing problem with simultaneous delivery and pickup," International Journal of Innovative Computing, Information and Control, vol. 9, no. 11, pp. 44974508, 2013.

[5] J. E. Bernard, L. Segel, and R. E. Wild:, "Tire shear force generation during combined steering and braking maneuvers," SAE Transactions 770852, 1977.

[6] H. Sakai, "Theoretical and experimental studies on the dynamics properties of tyres," International Journal of Vehicle Design, vol. 2, no. 3, pp. 335-372, 1981.

[7] G. Gim and P. E. Nikravesh, "Analytical model of pneumatic tyres for vehicle dynamic simulations-part 1: pure slips," International Journal of Vehicle Design, vol. 11, no. 6, pp. 589618, 1990.

[8] H. B. Pacejka and R. S. Sharp, "Shear force development by pneumatic tyres in steady state conditions: a review of modelling aspects," Vehicle System Dynamics, vol. 20, no. 3-4, pp. 121-176, 1991.

[9] H. B. Pacejka and E. Bakker, "The magic formula tyre model," Vehicle System Dynamics, vol. 21, pp. 1-18, 1993.
[10] D. J. Schuring, W. Pelz, and M. G. Pottinger, "A model for combined tire cornering and braking forces," SAE 960180, 1996.

[11] K. H. Guo and L. Ren, "A unified semi-empirical tire model with higher accuracy and less parameters," SAE Technical Paper 1999-01-0785.

[12] J. Svendenius and M. Gäfvert, "A semi-empirical dynamic tire model for combined-slip forces," Vehicle System Dynamics, vol. 44, no. 2, pp. 189-208, 2006.

[13] M. K. Salaani, "Analytical tire forces and moments with validated data," SAE Technical Paper 2007-01-0816, 2007.

[14] K. Guo, Y. Zhuang, D. Lu, S.-K. Chen, and W. Lin, "A study on speed-dependent tyre-road friction and its effect on the force and the moment," Vehicle System Dynamics, vol. 43, pp. 329340, 2005.

[15] K. Guo, N. Xu, D. Lu, and J. Yang, "A Model for combined tire cornering and braking forces with anisotropic tread and carcass stiffness," SAE International Journal of Commercial Vehicles, vol. 4, no. 1, pp. 84-95, 2011. 


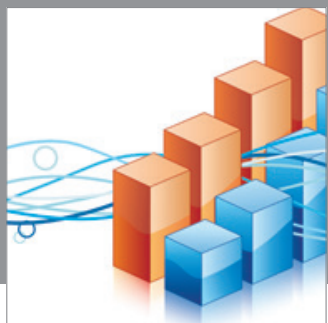

Advances in

Operations Research

mansans

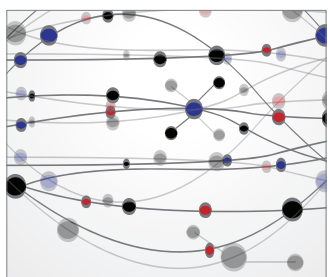

The Scientific World Journal
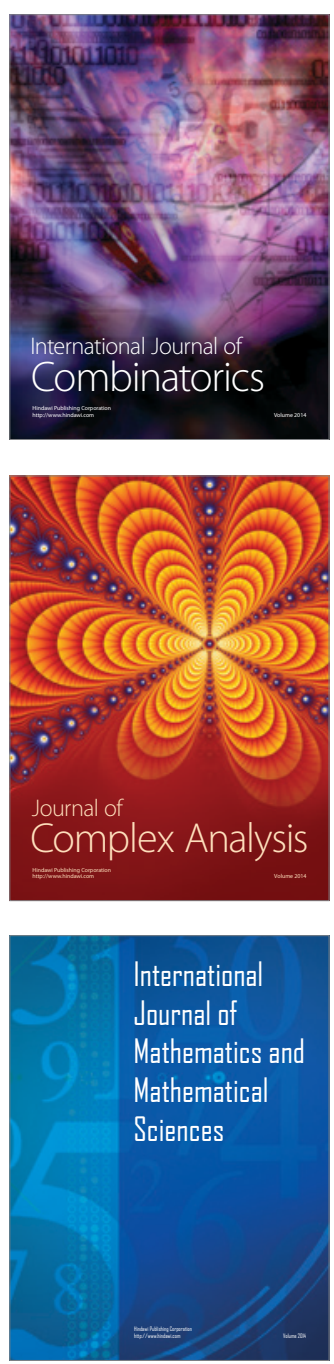
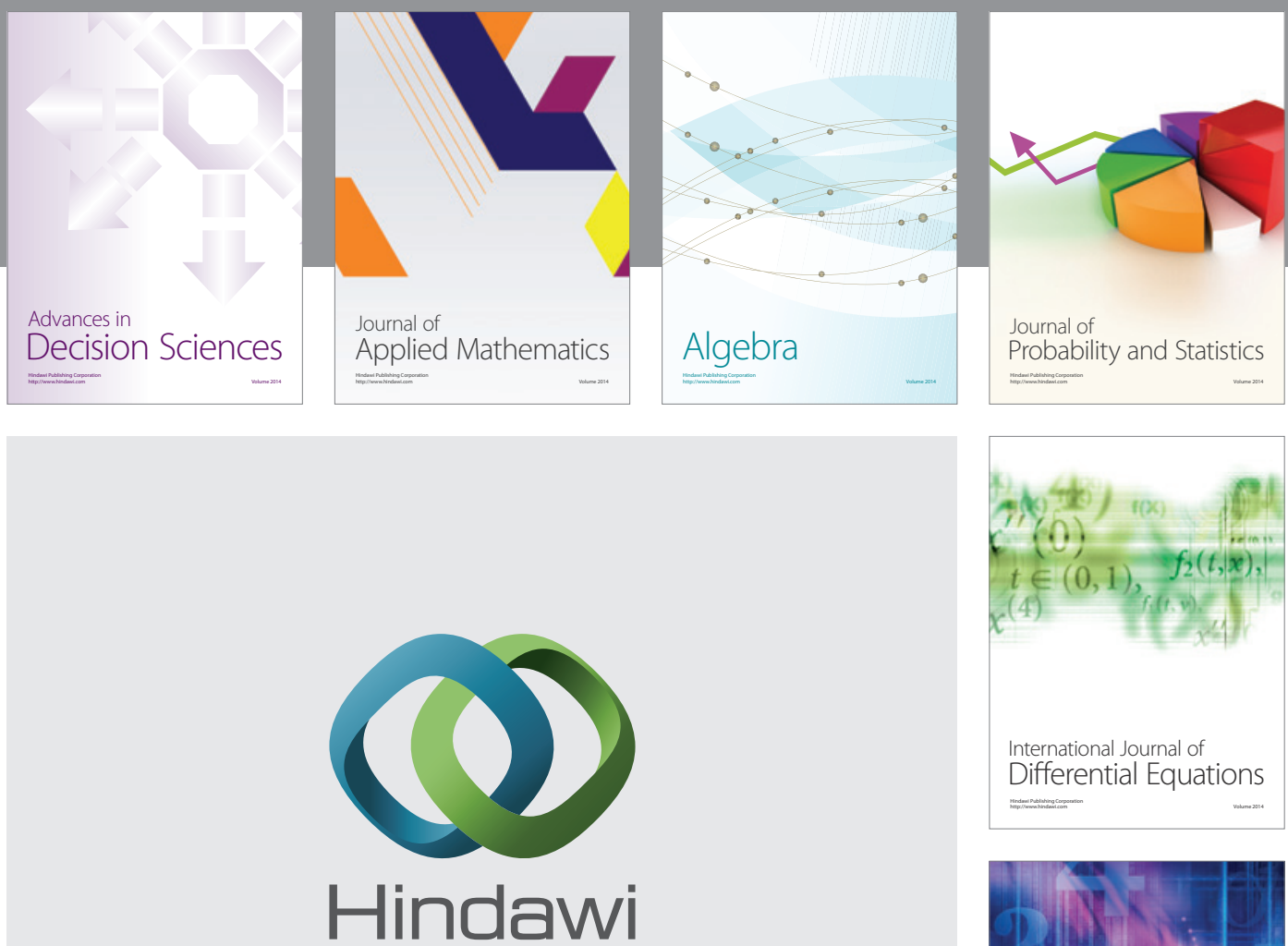

Submit your manuscripts at http://www.hindawi.com
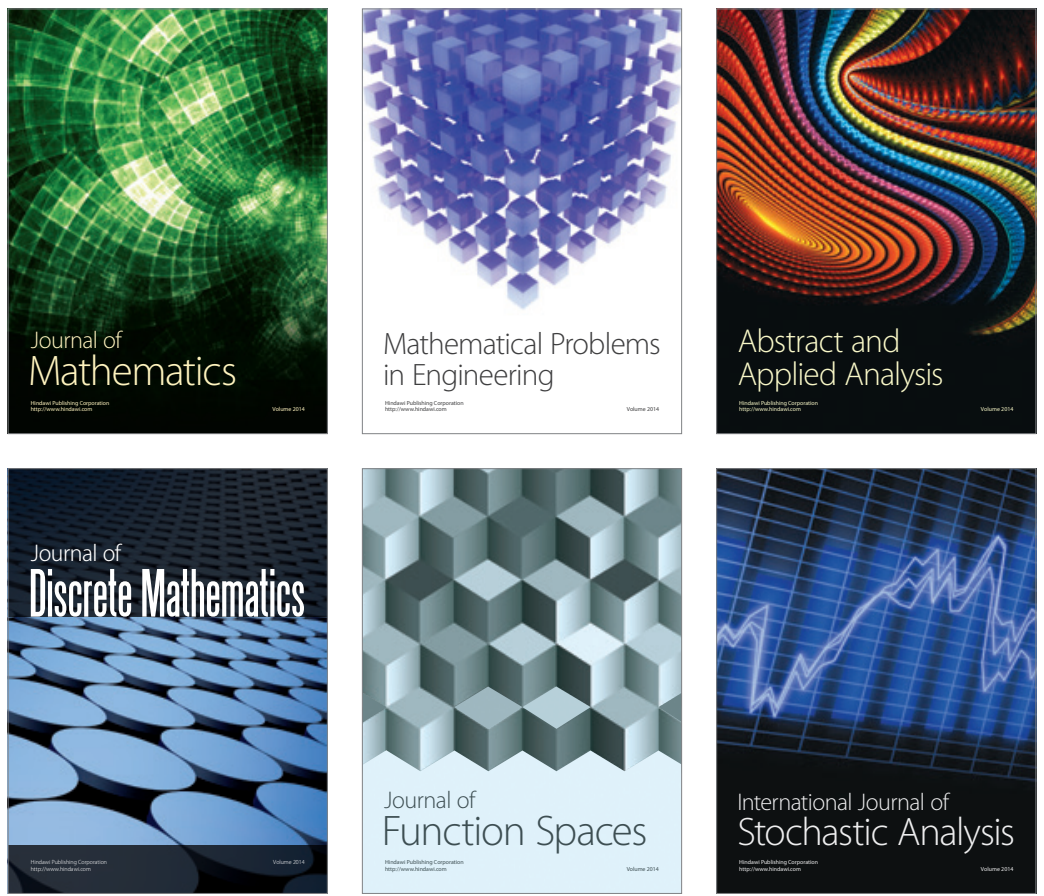

Journal of

Function Spaces

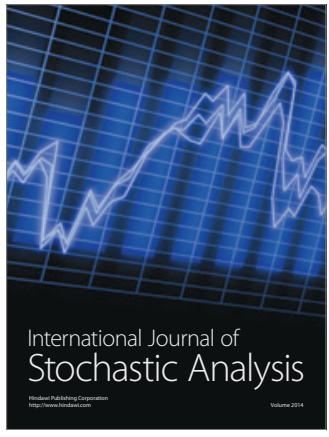

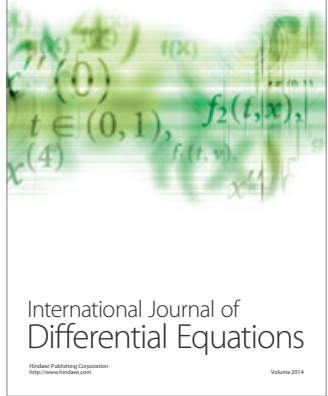
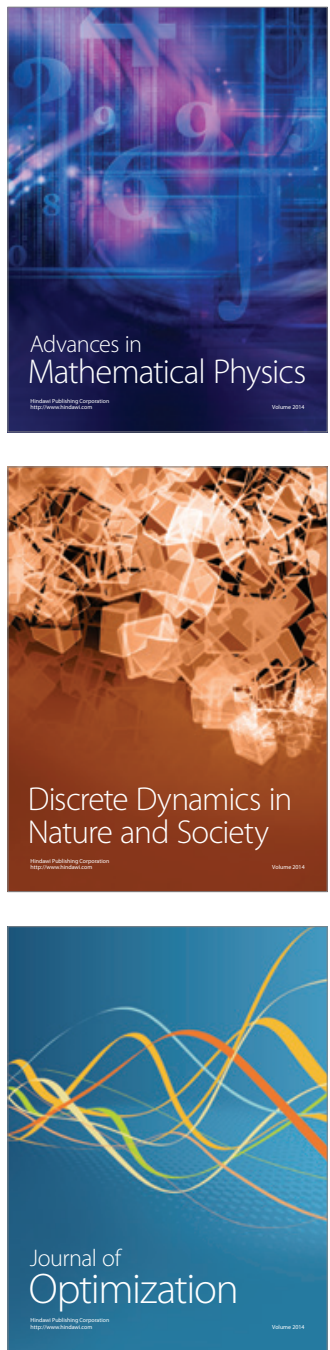